\title{
Conservando un símbolo: Tres siglos en la historia de la Puerta de Córdoba
}

\author{
Juan Antonio Arenillas Torrejón \\ Centro de Documentación del IAPH \\ Área de Patrimonio Mueble
}

La reciente restauración de la Puerta de Córdoba, en Carmona, ha posibilitado profundizar en el conocimiento histórico y artístico de tan emblemático monumento. La investigación documental paralela a los distintos procesos de estudio, análisis e intervención, ha permitido valorar las diversas actuaciones a las que se vio sometido el inmueble en su historia moderna y contemporánea. De este modo, se ha podido documentar con mayor precisión las obras realizadas entre 1608 y 1609, bajo la dirección de Martín Cardeno; se han analizado los proyectos realizados por los alarifes de Carmona, Gregorio González y Gaspar Peña, y el diseñado por Ignacio Moreno, en 1774 y 1775 respectivamente, y, por último, se han localizado nuevos datos respecto a la intervención de José Echamorro y definitiva configuración de la puerta. Tres siglos de historia de un edificio, en la que una constante, la existencia en el subsuelo de la propia puerta, de corrientes de agua, será la principal causa de los continuos desprendimientos de sillares y hundimientos, tanto en la puerta como en la muralla contigua.

\section{Obras y remodelaciones en el siglo XVI}

Escasas son las noticias que nos permiten conocer las distintas remodelaciones que sufrió la Puerta de Córdoba durante el quinientos. El primer dato de interés se refiere al acuerdo capitular de enero de 1525, por el que se encarga al jurado Luis de Rueda "faga la puerta de Cordova"!.

En el cabildo celebrado el II de agosto de 1544, se acuerda aprovechar "los sillares que estan a la Puerta de Cordova", para reparar la calzada "que se hunde" 2 . Esta noticia tiene cierto interés por cuanto el aprovechamiento de una serie de sillares localiza- dos en la puerta, parece indicar la posibilidad de ruina de la misma o en la muralla contigua. Por otra parte, su utilización en la calzada "que se hunde" podría hacer referencia al problema permanente de este inmueble, cual era las corrientes de agua subterráneas. En torno a |55|, se debieron realizar distintas labores según se desprende del cabildo celebrado el 26 de febrero, en el que los capitulares acuerdan pagar "lo gastado en la Puerta de Cordova", y continuar con las labores emprendidas 3 .

En el curso de la investigación sobre esta puerta carmonense no se han hallado más documentos sobre intervenciones durante el siglo XVI. Sí conocemos obras en otras puertas de la ciudad, como las efectuadas entre I 577 y I 579 en la Puerta de SeviIla, con motivo de la visita del rey Felipe II, para la que se levantó un arco de triunfo ornamentado con esculturas y "las armas reales y las de carmona", al modo de los realizados en la ciudad de Sevilla en la misma fecha ${ }^{4}$. Las puntuales actuaciones sobre las puertas de Carmona, realizadas en distintas fechas, parece indicar la falta de un proyecto general al modo del que se produjo en Sevilla en la segunda mitad del quinientos bajo la dirección del arquitecto Hernán Ruiz el Joven 5 .

\section{El siglo XVII y la intervención del cantero Martín Cardeno}

En 1595, el corregidor de Carmona don Antonio Maldonado expresaba en un escrito dirigido al cabildo, su preocupación por el estado general de las murallas de la ciudad "porque por muchas partes de ella se va cayendo y desboronando". Los capitulares acordaron hacer llegar al regidor don Alonso de Sotomayor, por entonces en la corte, sus inquietudes y la necesidad de que buscase medios económicos que permitiese abordar las obras 6 . Aunque no se citen explícitamente las puertas de la ciudad, creemos que debían presentar cierto estado de abandono. En el caso de la Puerta de Córdoba, en 1601 debía tener parte de sus muros y arco en ruina y caídos 


\author{
La puerta neoclásica adquirió con su nueva fisonomía, un mayor \\ protagonismo urbano, transformándose para el viajero que se \\ acercaba a Carmona desde el camino real, en punto focal y referente \\ de la ciudad.
}

sobre la calzada, como demuestra que en el cabildo celebrado el 30 de abril, los capitulares acordaran reparar "lo que se cayo de la puerta de cordoba y se faga muy bien fecho". Para la supervisión de las labores a realizar se nombraron a los regidores Jerónimo Barba y Bartolomé Bravo ${ }^{7}$.

Las corrientes de agua que corrían bajo el arco de la puerta de Córdoba fue, como ya se ha indicado, su constante y principal problema. La mayoría de las intervenciones efectuadas se debieron a esta circunstancia, llegándose en ocasiones al cierre del camino que se iniciaba en ella y, por tanto, a una de las vías principales de comunicación de la ciudad. En esta situación se encontraba a principios de I608, según se desprende de la petición presentada por el corregidor Diego Flores del Carpio Girón al cabildo, en la que manifestaba que "el camino por baxo la puerta de cordova se va reparando y quitando $\mathrm{El}$ arroyo que a corrido el camyno..." 8. Un mes más tarde, se le encargaba a don Martín de la Villa que urgentemente "faga la obra y la cabe e la ponga en perficion" 9 .

Cuando aún se ejecutaban estos trabajos, una de las torres que flanqueaba la puerta se desplomó, solicitando el corregidor a los capitulares que siendo la obra "tan grandiosa y bien parescida es justo que la villa mande quitar las piedras y fortalecer el muro por las partes que tuvieren necesidad...". Igualmente indicaba que había encargado a un escultor "fazer tres cudos en marmol blanco e un letrero en marmol negro ... con las letras de oro y renovar e aderesar lo que le falta a las estatuas de marmol..." 10. La obra fue encargada al maestro cantero Martín Cardeno, bajo cuya dirección estuvieron los canteros Hernán Pérez, Alonso Ruiz, Francisco de Peñaranda y Diego Muñoz, el albañil Alonso Pérez de Alaraz, el escultor Salvador Rodríguez, el cerrajero Juan Jiménez y los empedradores Juan de Castro y Luis de León.

Al margen de la propia labor de cantería, cuyo alcance no podemos conocer con exactitud, Cardeno

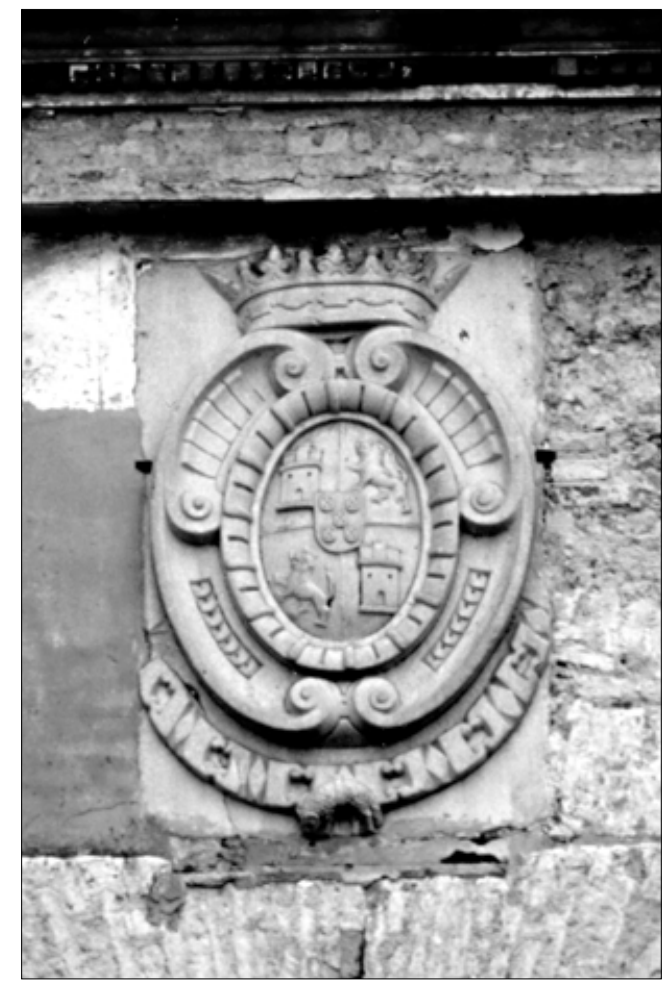

I. Salvador Rodríguez. Carmona. Puerta de Córdoba. Escudo real, 1608. "restauró" las esculturas, probablemente romanas que ornamentaban la puerta, una estatua del dios Apolo y una escultura de mujer, a la que tuvo que fijar la cabeza. Del documento firmado por Cardeno, certificando los distintos trabajos encargados al cerrajero Juan Ximénez, puede deducirse que las figuras debían estar fijadas a la pared, quizás en hornacinas y probablemente a ambos lados del arco "'. La puerta renovada con lenguaje clasicista, esculturas, escudos y lápidas con inscripciones fue la que debió ver Rodrigo Caro a su paso por la ciudad, en torno a 1634 12. Como elementos nuevos en el ornato, se incorporaron los escudos reales y de la ciudad, encargados al escultor sevillano Salvador Rodríguez, aún conservados, ${ }^{13}$ (Fig. I) y una serie de lápidas commemorativas. La dedicada a San Teodomiro, contiene los siguientes versos latinos: 


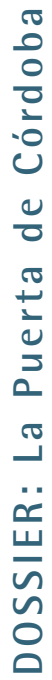

"NON QVIA CONSVRGAM SVBLIMIS AD AETERA CARMO

AVT MIHI SERTA FERAX SPICEA NECTAT AGER

NEC QVOD AB EIS VI SAT ME PHOSPHORVS ORIS

EFFEROR AVT CIVIS NOBILITATE MEI

ABS EGO TER FELIX DVPLICIS SPLENDORE PATRONI

SEV TEODOMIRI SIVE MATHEAE TVO"

que traducido dice:

"No porque en fuerte y levantada altura situada estoy, ó que de ricas mieses mis vegas me corones, yo me ufano. No porque el sol desde su Oriente alegre mis muros bañe, ó tanto me engrandezca de mis vecinos la nobleza antigua. Mas soy tres veces más dichosa y grande de dos patronos por la gloria y lustre; ó bien de Teodomiro, hijo mio, ó bien Mateo, apóstol por el tuyo" 14.

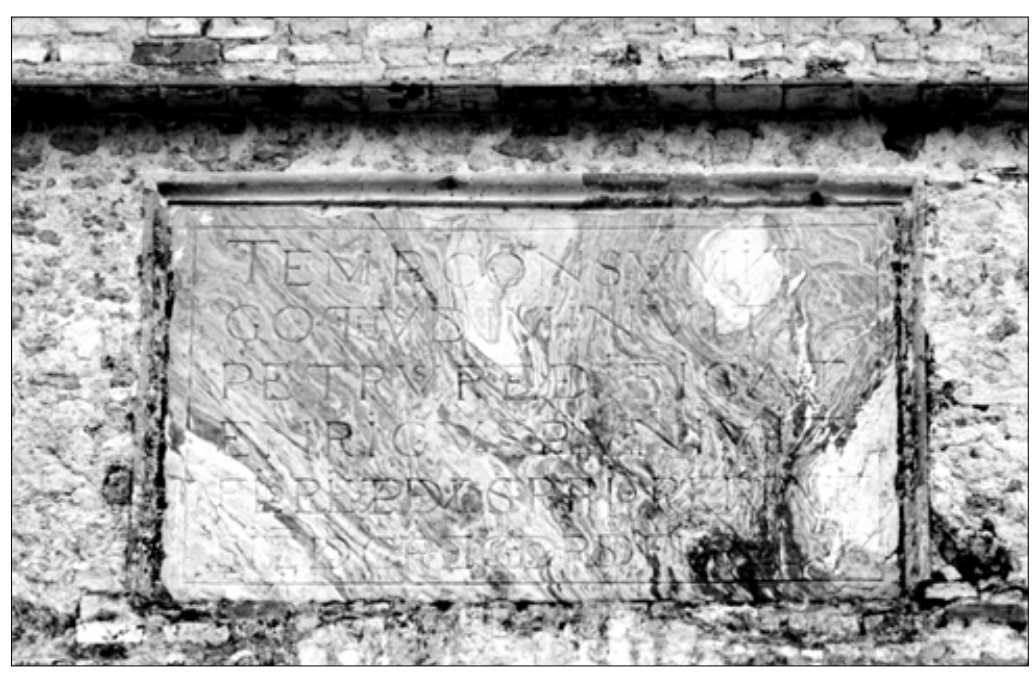

2. Carmona. Puerta de Córdoba.

Lápida commemorativa, |608-1609.
Según se recoge en "El curioso carmonense ocioso divertido", en "el frente interior que mira a la ciudad hay otros versos latinos en una lápida con capiteles que sirven de coronación a la puerta". Resulta muy interesante que se reproduzca el remate de la fachada oeste, de tal manera que nos podemos hacer una idea de su fisonomía en esas fechas ${ }^{15}$. La inscripción dice:

"TEMP. CONSVMIT

GOTHVS DIMINVIT.

PETRVS REEDIFICAVIT

ENRICVS PUNIVIT.

FL ${ }^{\circ}$ REP DEVL SCARPIO REPARAVIT

SIENDO COREGIR D ${ }^{\circ}$ FLORES CAPO 1609". (Fig. 2.)

No cabe duda de que la llegada de la reliquia de San Teodomiro a la ciudad, en manos del padre fray Rodrigo de Quintanilla y el alférez mayor de Carmona don Lázaro de Briones, procedente de Córdoba, fue acogida por los carmonenses con gran entusiasmo, haciendo que Carmona se engalanara con "colgaduras, arcos triunfales y demás aparato... correspondiente a tan magnífica función". Esta circunstancia debió ser la principal causa de la renovación de la Puerta de Córdoba, ya que por ella entró la reliquia en la ciudad, y en ella se inició el recorrido solemne del cortejo.

Desde el punto de vista arquitectónico, desconocemos el alcance de la intervención de Cardeno. Pudo limitarse a la reconstrucción de la torre, la intervención sobre las esculturas y su colocación en la puerta, así como el encargo y supervisión de la labor de las lápidas y los escudos. Sin embargo, el hallazgo de distintos elementos arquitectónicos durante la reciente intervención, como restos de bolas de piedra serpentina o posibles basamentos, pudieran deberse al proyecto efectuado en 1608 , lo que nos llevaría a pensar en un posible cambio estético en la puerta, sobre todo, en su fachada interior, a la que se pudo dotar del lenguaje manierista propio de la época. Se configura entonces como elemento clave y símbolo de la ciudad, manteniendo la visión exterior de solidez propia de su integración en la fortaleza, y esa imagen interior urbana, renovada, donde arquitectura y ornato ofrecerían una perfecta armonía.

Sólo tres años más tarde, en 1611, la puerta tuvo que repararse de nuevo ${ }^{16}$. Otras noticias referentes a obras en la puerta durante el siglo XVII, se documentan en 1648, cuando el procurador mayor don Juan Teodomiro de Cabrera expresa en un escrito dirigido al cabildo la "ruyna muy grande robada con las corrientes de las aguas de suerte que siendo como es el paso comun destos reynos no se puede trajinar con coches ni literas..." 17. Por último, en 1678, el mal estado de conservación de la puerta había obligado a su cierre, y en el cabildo celebrado el 23 de junio, los capitulares se quejaban de dicha situación "por ser de mucho comercio y entrada", y acordaron abrirla de nuevo y emprender las labores que fueran necesarias 18 .

La historiografía tradicional ha recogido que la actual fisonomía de la puerta se debía a una reedificación efectuada en tiempos del rey Carlos II, concretamente en 1668, por el maestro Alonso Rodríguez, en base a una lápida commemorativa localizada en el monumento. La lectura detenida de la inscripción indica que no se realizaron obras sobre la puerta, sino que se efectuaron en la calzada o Camino Real que unía la ciudad con la villa de Madrid. Además, la fecha de dichas labores no fue 1668, sino que las mismas abarcaron el periodo comprendido entre I688 y |69|. Para tal efecto, Rodríguez realizó una planta de la obra que entregó a don Diego Luis de Rueda y Mendoza, diputado de las calzadas del camino real y de la puerta de Córdoba. Para las distintas labores, Rueda expresaba que "no tenia inconbeniente que los sillares y Piedras Grandes que de ruinas antiguas an caido y estan enterradas en los cortinales y tierras labradas ... y asimismo Las que an caido en el aroio de las alberguillas y de la puerta de cordoba que embarasan el paso a las aguas" 19. 


\section{El proyecto de renovación del Camino Real por los alarifes Gregorio González y Gaspar Peña}

El 19 de mayo de 1773, los maestros alarifes Gregorio González y Gaspar Peña, evalúan el costo de distintas obras que debían hacerse en "el camino de la calzada y Puentte como assimismo ademas camino Real hasta el Rio de corbones". Para tal efecto realizaron en 1774, un "Mapa y Perfil" en el que recogían el camino real desde la Puerta de Córdoba hasta el puente ${ }^{20}$

Interesa de este proyecto, el dibujo de la fachada exterior de la puerta. De su arquitectura cabe resaltar el arco de medio punto flanqueado por pilares rematados en pirámides y bolas, así como la altura del remate amurallado a un solo nivel con las torres laterales, culminado en merlones piramidales. No se recoge en el proyecto de los alarifes ninguna posible intervención sobre la puerta, por lo que, puede decirse que es fiel reflejo de su imagen exterior en 1774. (Fig. 3)

\section{El proyecto de Ignacio Moreno}

En 1775, fue requerido Ignacio Moreno, maestro mayor de los Reales Alcázares de Sevilla, para que diese su parecer sobre las obras necesarias en el Camino Real. Moreno levantó un plano que comprendía distintos alzados entre los que se encontraba la Puerta de Córdoba. A diferencia del proyecto de González y Peña, el arquitecto introduce distintas labores a realizar. En su informe, elaborado el I de junio de 1775, indica que la torre norte estaba arruinada y "desplomada y quebrada de diferentes partes", recomendando su inmediata demolición. En su proyecto, Ignacio Moreno planteaba "labrar la expresada torre Ruinosa de Canteria por lo esterior y en lo ynterior debera ser masiso de mamposteria hasta el medio de su altura", la cual debía poseer la misma altura que la torre sur. Proponía igualmente, reducir la altura del tramo de puerta comprendida entre las dos torres, unas tres varas, como muestra el diseño, aunque volvería a incorporar el almenado superior "para su adorno, hermosura y acompañamiento al excudo de Armas que esta sovre el mismo Arco" 21.

El diseño aportado por Moreno del alzado de la puerta de Córdoba en su fachada oriental, muestra con claridad como quedaría tras la remodelación propuesta. Puede apreciarse respecto al dibujo de los alarifes carmonenses pérdida de altura en el cuerpo central, el almenado con el escudo al centro, y dos arbotantes en los extremos contra las torres. El vano central, de medio punto, quedaría flanqueado por pilastras adosadas rematadas en pirámides y bolas. ${ }^{22}$ En el cabildo celebrado el día 17 de noviembre de 1775, se leyó una carta de don Marcos de Ubiurra dirigida a don Manuel Becerra, en la que indicaba que tras el análisis de ambas propuestas, se había decantado por la presentada por el arquitecto Ignacio Moreno ${ }^{23}$. (Fig. 4)
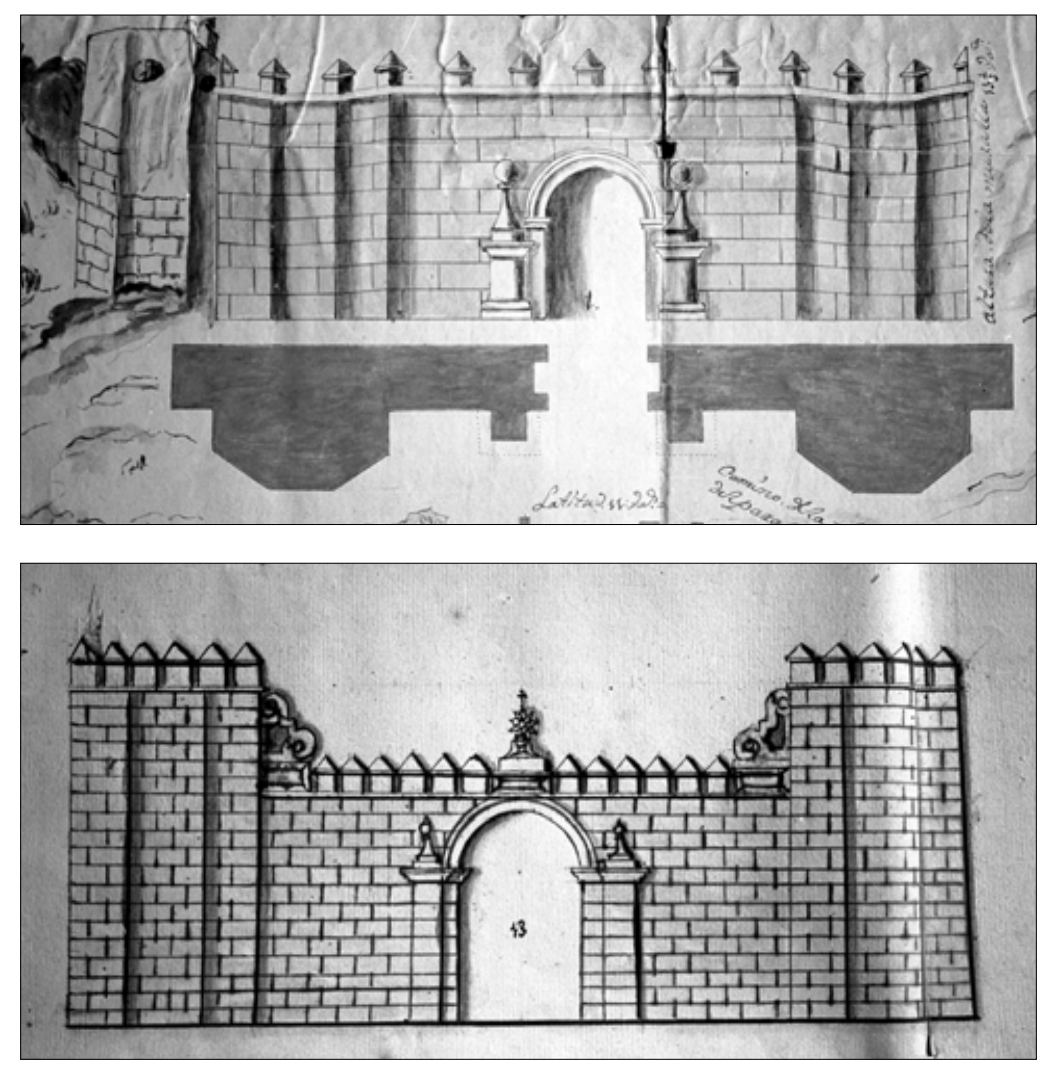

\section{José Echamorro y la puerta neoclásica}

El último gran proceso constructivo que dio la actual fisonomía a la Puerta de Córdoba, se inicia en 1790. En el cabildo municipal celebrado el 15 de julio, se leyó una carta del intendente José de Avalos en la que se señalaba como el Real y Supremo Consejo de Castilla había decidido prorrogar "el Arvitrio que antes havia disfrutado de dos mrs en quartillo de vino", con la finalidad de destinarlo "a la reparacion del Arco que llaman la Puerta de Cordova la de las Casas Capitulares en lo correspondiente a la pieza del Archivo Escribania de Cavildo como tambien para la Escuela de primeras Letras" 24. La obra se encargó al arquitecto José Echamorro, presentando el correspondiente pliego de condiciones un año más tarde. En febrero de |79|, los capitulares acuerdan acometer en primer lugar, las labores necesarias en las Casas Capitulares 25

Según se desprende de un informe realizado por Lorenzo José Domínguez y presentado al cabildo municipal el 4 de julio de 1800, José Echamorro realizó dos proyectos, iniciándose las obras en 1796. El primero de ellos debió comprender la labor de las dos fachadas, así como "sus dos castillos colaterales" o torres, incluyendo la balaustrada. Sin embargo, en el transcurso de las obras, el arquitecto planteó la edificación del ático, "una Piesa cubierta evitable sobre el mismo Arco", así como la realización de una "Madrona o desagüe por devajo de los Muros que sostienen dicho Arco", con el fin de dar una solución definitiva al problema de las corrientes de agua subterráneas que tanto daño habían causado a la puerta en el
3. Proyecto de Gregorio González y Gaspar Peña. Detalle.

4. Proyecto de Ignacio Moreno. Detalle. 


\section{CRONOLOGÍA DEL MONUMENTO}

1504

Se produce en Carmona un fuerte terremoto que afectó al sistema amurallado de la ciudad, incluyendo sus puertas.

1525

enero / 2

El cabildo municipal ordena al jurado Luis de Rueda "que

faga la puerta de Cordova...”, no especificándose la

dimensión de la obra.

1544

Se reaprovechan una serie de sillares probablemente de la

agosto / 11 puerta, para reparar la calzada real.

1551

febrero / 26

Se realizan una serie de labores en la puerta, sin especificarse

\section{1}

abril / 30

Parte de la puerta se encontraba en ruina y se ordena a los regidores Jerónimo Barba y Bartolomé Bravo "se repare lo que se cayo de la puerta de cordoba y se faga muy bien fecho". En la misma debieron intervenir el maestro carpintero Diego de Morales que realiza una puerta y el albañil Pero Hernández.

1608

febrero / 8

Se hicieron diversos trabajos para subsanar los problemas

\section{8}

marzo / 3

1608

marzo / 29

1611

febrero / 25 Se acometen distintos reparos.

1648

octubre / 13

1678

junio / 23
El procurador mayor de la ciudad Juan Teodomiro de Cabrera

Se encarga a Martín de la Villa supervise los trabajos que debían realizarse en la puerta referente a las corrientes de agua subterráneas.

Se desploma una de las torres que flanqueaba la puerta y se inician con urgencia las labores de reconstrucción ante la inminente llegada de la reliquia de San Teodomiro procedente de Córdoba. La obra estuvo bajo la dirección del cantero Martín Cardeno. Al escultor Salvador Rodríguez se encargan tres escudos en mármol blanco. Además se hicieron distintas lápidas commemorativas con textos latinos, y se restauraron las dos esculturas de mármol, una de Apolo y otra de una figura de mujer, para su ubicación definitiva en la puerta. Las obras se concluyeron en 1609.

y Navarra comunicó al Ayuntamiento "que la puerta cordoba esta con una ruyna muy grande robada con las corrientes de las aguas de suerte que siendo como es el paso comun destos reynos no se puede trajinar con coches ni literas da cuenta a la siudad para que ponga el remedio nesesario". Los capitulares acordaron realizar los reparos necesarios.

La Puerta de Córdoba se encontraba cerrada y los vecinos piden que se abra "por ser de mucho comercio y entrada". Se acuerda abrirla y repararla. transcurso de los siglos. ${ }^{26}$ Aunque tradicionalmente se venía recogiendo la fecha de 1800 como la de finalización de la obra, el informe citado o el pago que se hace al maestro aparejador de la obra y hermano de nuestro arquitecto, Juan Echamorro, con posterioridad a esa fecha, corrobora que aún se realizaban trabajos en los primeros años del siglo XIX ${ }^{27}$.

José Echamorro nace en Carmona el 18 de marzo de 175।. Hijo de Antonio Chamorro, alarife de la propia ciudad, y de Catalina Manzano, debió aprender el oficio junto a su padre, familiarizándose con el lenguaje arquitectónico propio de la época, así como con los diversos instrumentos necesarios para el dibujo y la traza. ${ }^{28}$ En 1786 es nombrado maestro mayor de obras de la ciudad de Sevilla, tras la renuncia de Félix Caraza, después de presentarse a un concurso al que concurrieron los más prestigiosos arquitectos, maestros de obras y alarifes. ${ }^{29} \mathrm{~A}$ tal efecto, Echamorro adjuntó un certificado firmado por Lucas Cintora como Director General de la Real Escuela de las Tres Nobles Artes: Pintura, Escultura y Arquitectura, en el que se recoge: "D. Joseph Echamorro ha cursado las tareas del Dibujo en la Clase de Arquitectura, desde su establecimiento, con infatigable desvelo, y assi mismo ha hecho un acto de oposición a los Premios Generales, que se celebraron en el Año de Mil Setecientos ochenta y dos". Además de estos estudios, Echamorro estuvo dos años en la Academia de San Carlos de Valencia, asistiendo también a clases de matemáticas en el Colegio de San Hermenegildo de Sevilla ${ }^{30}$. A pesar de ello, nunca obtuvo el título de arquitecto, como ha documentado Suárez Garmendia 31 .

Aunque no se ha definido con profundidad la figura de este arquitecto en el contexto de la arquitectura sevillana, sí podemos decir que tuvo una intensa actividad, proyectando edificios de nueva construcción, participando en remodelaciones importantes e incluso diseñando obras de carácter ornamental. En la relación de méritos que presentó el arquitecto para optar a la maestría mayor de obras de la ciudad de Sevilla, indica su participación en la construcción de la Iglesia de San Bartolomé, las dos naves colaterales de la Iglesia de los Remedios, ambas en la capital hispalense, y el pórtico y la fachada este de la carmonense parroquia de Santa María. Además cita la realización de un cancel de yesería para el convento de carmelitas descalzos de Sanlúcar de Barrameda, la ejecución de una serie de planos de un cuartel de caballería que no llegó a construirse, y otras labores de infraestructura en las alcantarillas de la Torre del Oro y San Bernardo y en el camino de Castilleja de la Cuesta 32.

Por orden cronológico, la primera obra documentada se refiere a la edificación del "Puente Chico" sobre el río Salado en Tarifa y la reconstrucción de una mina de agua en Osuna, ambas en 1787.33 Entre 1788 y 1802 interviene en la Iglesia de San Pedro de Peñaflor, donde fortalece los pilares del interior del templo, cambiando el orden arquitectónico de corintio a dórico, e incorporando un friso con triglifos y metopas. Al exterior proyectó el cuerpo 
Entre 1790 y $179 \mid$ realiza el primer proyecto de la Puerta de Córdoba en Carmona, así como la remodelación proyectada en las Casas Capitulares, en la misma ciudad ${ }^{35}$. En 179 | es nombrado maestro mayor diocesano, proyectando entonces la Cilla del Cabildo de Carmona, para la que presentó dos diseños, fechados en 1791 y 1794 36. En 1795 reedifica la Puerta de la Macarena de Sevilla, 37 y un año más tarde, concluye el cuerpo de campanas de la sevillana Iglesia de San Bartolomé, proyectada en 1780 por Antonio de Figueroa 38. Suárez Garmendia ha documentado como obra de Echamorro, la Iglesia de Santiago de Utrera, para la que debió dar trazas en 1795, concluyéndose su construcción cuatro años más tarde ${ }^{39}$. En 1800, se encarga de la ejecución material de la Iglesia de San Ildefonso de SeviIla, siguiendo los diseños de Julián Barcenilla 40. Entorno a 1812 , realiza un informe sobre el estado en que había quedado la Cartuja de las Cuevas tras la presencia francesa en el monasterio ${ }^{41}$. La última obra documentada de Echamorro se refiere a la restauración que en colaboración con Manuel Cintora realizó en 1819, en la portada de la Casa de la Moneda. ${ }^{42}$ También se le han atribuido las fachadas laterales de la Iglesia de Santa María de la Mesa de Utrera y el templo de la Encarnación de Olvera ${ }^{43}$

\section{Análisis formal y estilístico}

José Echamorro está considerado como el primer arquitecto neoclásico sevillano, cuyo estilo supera el eclecticismo académico, dotando a su arquitectura de la ortodoxia y sobriedad del lenguaje neoclasicista ${ }^{44}$. Sin embargo, analizando su producción arquitectónica puede apreciarse la pervivencia de ciertos elementos que podríamos considerar aún como barrocos, caso de la decoración de la portada proyectada para la Cilla del Cabildo de Carmona o el uso constante de vanos con orejetas.

Como ya se ha indicado con anterioridad, Echamorro realizó su primer proyecto para la Puerta de Córdoba, entre 1790 y 1791, si bien las obras no se iniciaron hasta 1796. En él se contemplaba configurar de nuevo la puerta en sus dos fachadas, así como la ejecución de las torres que la flanquean. La fachada oriental presenta arco de medio punto con enjutas decoradas, flanqueado por dobles columnas toscanas sobre basamentos y pilastras almohadilladas. Los intercolumnios se ornamentan con una ventana fingida entre pilastras rematada por friso con triglifos y metopas y frontón triangular. Sobre éste, óculo cegado. En los extremos, columnas del mismo orden arquitectónico. Como culminación, se dispone un friso con triglifos y metopas, como corresponde a la concepción de una fachada de orden dórico o toscano. Remata el conjunto en balaustrada ciega sólo interrumpida por pedestales rematados en jarras, coincidentes con la disposición inferior de las columnas. En el centro y como remate de la fachada, Echamorro dispuso un ático con vano central adintelado, pilastras almohadilladas y frontón triangular

\section{CRONOLOGÍA DEL MONUMENTO}

1688

septi / 10

Se realiza la obra de la calzada real, la cual trazó y dirigió el sillares y piedras grandes que estaban en la puerta.

\section{3}

mayo / 19

Don Diego Luis de Rueda y Mendoza como diputado de las

1774 calzadas del camino real, solicita se reparen y se presenten proyectos para su realización.

Los maestros alarifes de Carmona, Gregorio González y Gaspar Peña realizan "un Mapa y Perfil" del camino, la calzada real y puente, en el que ofrecen un alzado de la fachada oriental de la puerta.

1775

junio / 1

El arquitecto Ignacio Moreno presenta su proyecto sobre las obras de infraestructura necesarias en las calzadas, camino y puente. Incorpora además un alzado de la puerta en la que introduce importantes variantes como la disminución en altura de la puerta en relación a las torres o la colocación de contrafuertes apoyados en ellas.

1775 Marcos de Ubiurra comunica a Manuel Becerra que el noviem /17 proyecto aprobado es el del arquitecto Ignacio Moreno.

1790

julio / 15

El Intendente de los Cuatro Reinos de Andalucía, don José de Avalos, comunica a los capitulares que el Real y Supremo Consejo de Castilla, había concedido a Carmona la prórroga del arbitrio de dos maravedís sobre cuartillo de vino, con la finalidad de acometer obras en la Puerta de Córdoba, el archivo de las Casas Capitulares y la Escuela de Primeras Letras. Se encargó el proyecto al arquitecto José Echamorro y se nombraron como diputados de la obra a Juan Caro Locella y Lorenzo José Domínguez.

1791

Echamorro presenta pliego de condiciones para las obras,

febrero / 8 acordándose empezar por las Casas Capitulares. El primer proyecto de Echamorro no contemplaba la construcción del ático.

1796

Inicio de las obras de la puerta por el aparejador Juan Echamorro, hermano del arquitecto.

Según un informe elaborado por Lorenzo José Domínguez, en esas fechas aún no se había concluido la obra de la puerta, por haber ampliado el proyecto Echamorro.

Esa ampliación consistió en la realización de "una Piesa cubierta avitable sobre el mismo Arco"y "una Madrona ó desague por devajo de los Muros".

$1802-1803$

Se pagan a Juan Echamorro, aparejador de la obra 3.744 reales y 8 maravedís. 


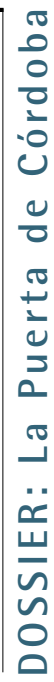

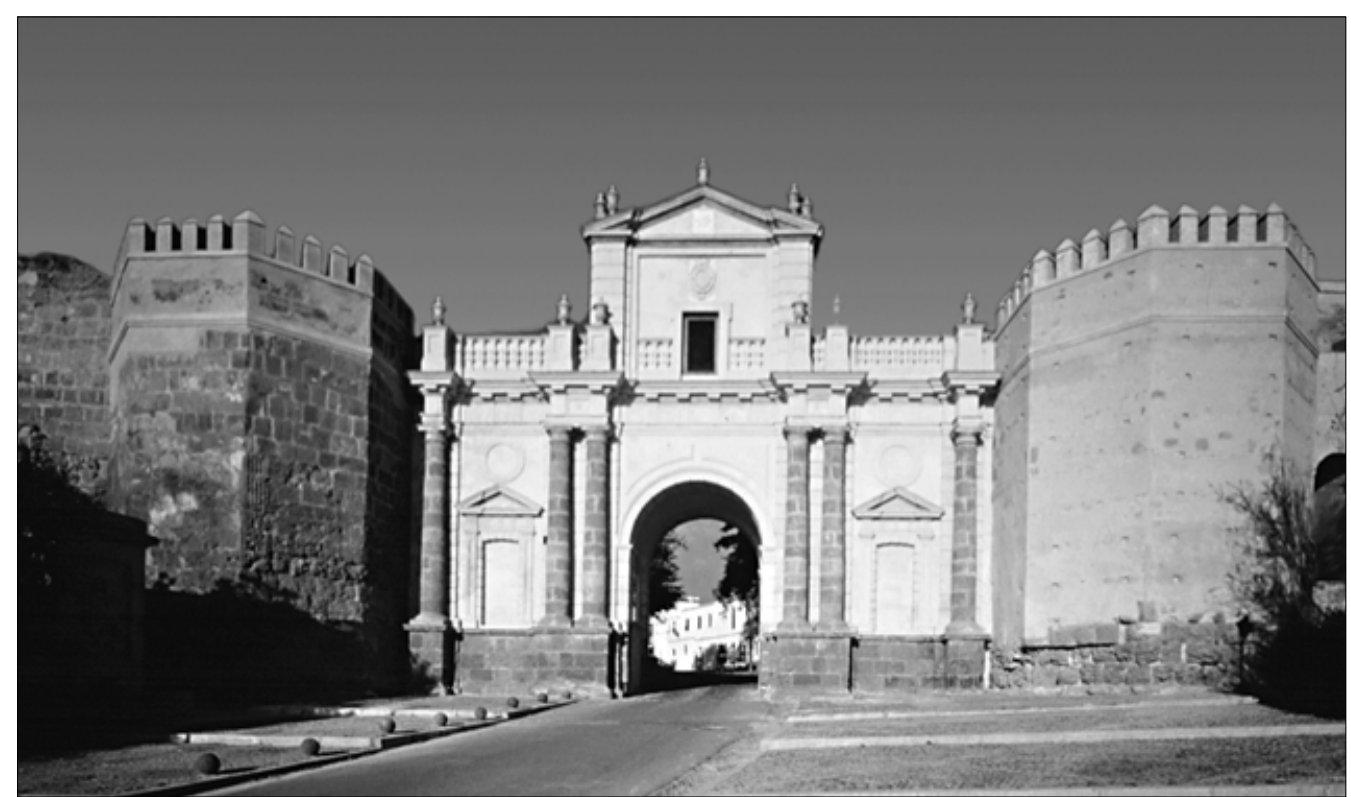

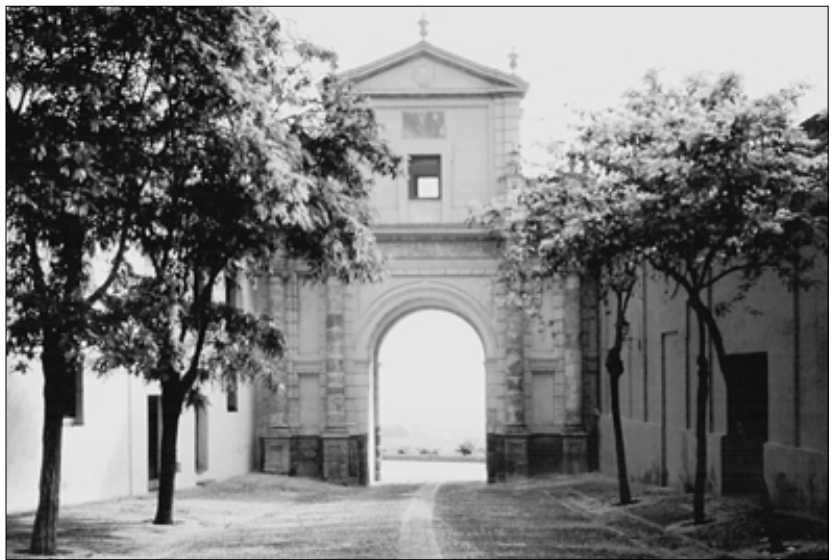

5. José Echamorro. Carmona. Puerta de Córdoba. Fachada exterior.

6. José Echamorro. Carmona. Puerta de Córdoba. Fachada interio

7. José Echamorro. Carmona. Puerta de Córdoba. Fachada interior. Detalle.

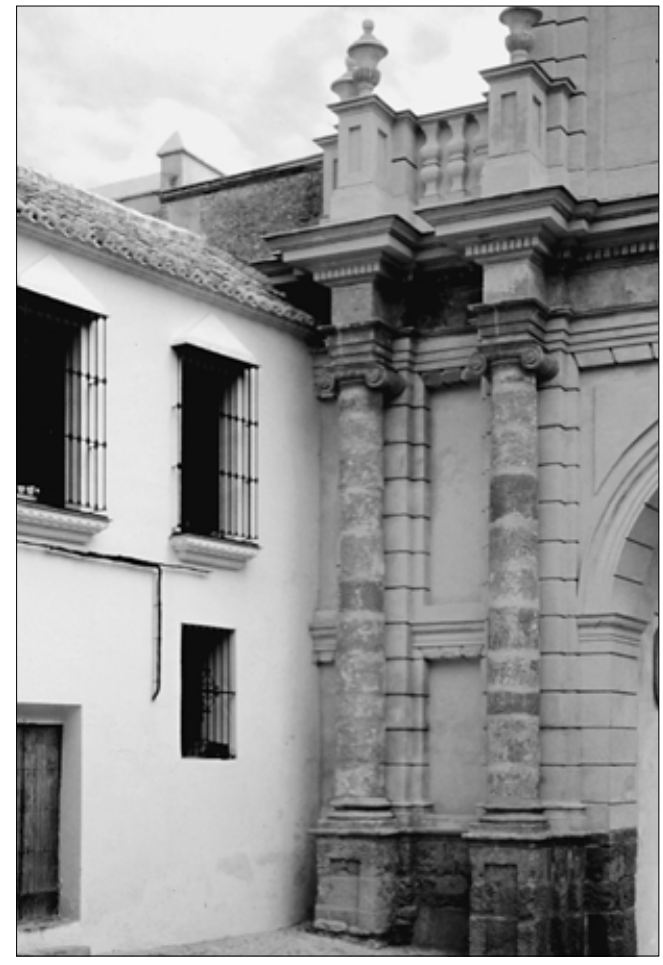

como remate. (Fig. 5) La fachada occidental, por su parte, presenta dobles columnas jónicas sobre basamentos apareciendo en los intercolumnios registros rectangulares. Culmina esta fachada en un entablamento liso, como corresponde a la concepción de una arquitectura de orden jónico. Aunque Echamorro transformó profundamente esta fachada, su articulación nos hace sospechar la posibilidad de que el arquitecto se viera mediatizado por estructuras preexistentes que le hicieran trazarla con menor libertad que la oriental. En este sentido, hay que recordar que la remodelación realizada entre 1608 y 1609, afectó fundamentalmente a la puerta interior, y que en los dibujos que realizaron Gregorio González, Gaspar Peña e Ignacio Moreno, en 1774 y 1775 , donde mostraban la puerta exterior, ésta no parecía poseer elementos importantes como para ser tenidos en cuenta por nuestro arquitecto, a la hora de plantear la nueva traza. (Fig. 6)

La puerta neoclásica adquirió con su nueva fisonomía, un mayor protagonismo urbano, transformándose para el viajero que se acercaba a Carmona desde el camino real, en punto focal y referente de la ciudad. A esto se unía un nuevo sentido estético, sobrio y monumental, propio de la arquitectura neoclásica. Este sensación de sobriedad se veía atemperada en la fachada occidental, con el empleo del orden jóni$\mathrm{co}$, de mayor esbeltez. A todas estas circunstancias hay que añadir, el respeto que mostró el arquitecto al reaprovechar los escudos y lápidas commemorativas que se habían realizado en 1608, desconociéndose en la actualidad si las dos esculturas colocadas en esa fecha, permanecían en la puerta cuando Echamorro ejecuta su proyecto. (Fig. 7)

En el contexto de la producción de Echamorro, la puerta de Córdoba en Carmona, presenta numerosos paralelos respecto a otras obras realizadas por el arquitecto. Se le atribuye, la fachada exterior de la desaparecida Puerta Nueva o de San Fernando en Sevilla, la cual debió realizar entre 1788 y los prime- 
ros años del siglo XIX ${ }^{45}$. Por lo tanto, ambas puertas son contemporáneas. (Figs. 8 y 9)

Al igual que la Puerta de Córdoba, la sevillana está concebida a modo de arco triunfal, con un solo vano central de medio punto. En ambas, Echamorro emplea columnas toscanas sobre basamentos, pilastras almohadilladas y una balaustrada ciega como remate. Pero también se introducen variantes, en cuanto a la eliminación del friso con triglifos y metopas o la incorporación en los intercolumnios de molduras similares a las utilizadas por Van Der Borcht en la fachada de la vecina Fábrica de Tabacos, hoy Universidad. De gran interés es la falta del ático en la puerta sevillana, por cuanto nos podemos imaginar como debió ser el primer proyecto de Echamorro para la Puerta de Córdoba en Carmona ${ }^{46}$.

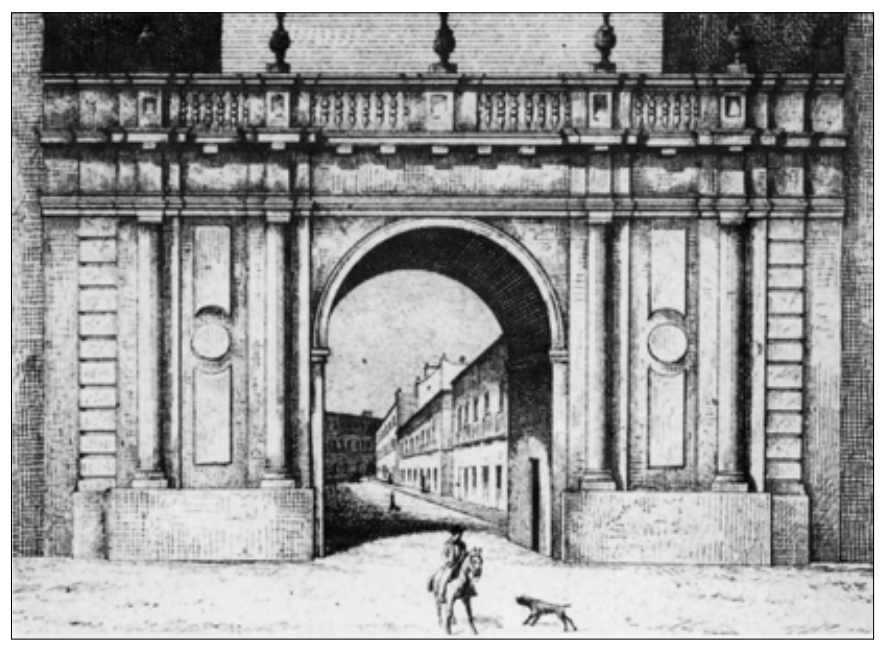

8. Sevilla. Puerta Nueva o de San Fernando. Fachada exterior. B. Tovar, 1878.

9. José Echamorro. Carmona. Puerta de Córdoba. Fachada exterior
El análisis comparativo de la puerta de Carmona con otras obras de Echamorro, permite establecer una serie de constantes estilísticas, propias del lenguaje arquitectónico al uso en la época, así como reiterativas en su obra. De este modo podemos hablar del uso de columnas sobre basamentos, como en los cuerpos de campanas de las iglesias de San Pedro de Peñaflor y, San Ildefonso y San Bartolomé de Sevilla. De la torre de este último templo sevillano pudo tomar la solución dada a los intercolumnios de la fachada oriental de la Puerta de Córdoba, con el uso de vanos adintelados rematados con frontón triangular y óculo. La utilización del orden dórico o toscano incorporando friso con triglifos y metopas, y el orden jónico con entablamento liso, muestra el conocimiento que Echamorro tenía sobre la tratadística clásica y los modelos arquitectónicos neoclásicos. Otra constante en la obra de José Echamorro es la incorporación de pilastras almohadilladas, que pueden observarse en la fachada exterior de la puerta, y a la que recurrió para la articulación de la fachada de la Cilla del Cabildo de Carmona, edificio cuya construcción debió correr paralela a la de la Puerta de Córdoba. 
I. HERNÁNDEZ DÍAZ, José; SANCHO CORBACHO, Antonio; COLLANTES DE TERÁN, Francisco: Catálogo Arqueológico y Artístico de la Provincia de Sevilla, vol. II, Sevilla, 1943, pp. 213 y 274. Es probable que el terremoto acaecido en 1504, afectase a la puerta de Córdoba, como a otras de la ciudad, y que se encontrara arruinada en esos momentos, FERNÁNDEZ, Manuel: Historia de la ciudad de Carmona, Reimpr. Sevilla, 1996, p. 302.

2. HERNÁNDEZ DÍAZ, José; SANCHO CORBACHO, Antonio; COLLANTES DE TERÁN, Francisco: Catálogo... Op. cit. p. 274.

3. Ibídem, pp. 213 y 274

4. FERNÁNDEZ , Manuel: Op. cit. p. 306. Las obras fueron realizadas por el cantero Juan Rodríguez, HERNÁNDEZ DÍAZ, José; SANCHO CORBACHO, Antonio; COLLANTES DE TERÁN, Francisco: Op. cit. pp. 273-274. En relación a las puertas levantadas en Sevilla con motivo de la presencia del rey, cabe resaltar la iconografía representada en uno de los arcos, en el que como remate figuraba el Dios Apolo en el Monte Parnaso, CABRA LOREDO, Ma D.: Iconografía de Sevilla. 1400 1650, Madrid, 1988, pp. 92-93.

5. Respecto a las puertas de Sevilla véase, MORALES, Alfredo J:: Hernán Ruiz el Joven, Madrid, 1996, pp. I17-1 I8, y JIMÉNEZ MAQUEDA, Daniel: Las Puertas de Sevilla. Una aproximación arqueológica, Sevilla, 1999.

6. HERNÁNDEZ DÍAZ, José; SANCHO CORBACHO, Antonio; COLLANTES DE TERÁN, Francisco: Op. cit. p. 273.

7. Archivo Histórico Municipal de Carmona (A.H.M.C.). Actas Capitulares. Libro 44. Cabildo celebrado el día 30 de abril. Aunque desconocemos los detalles de esta obra, sí sabemos que se le pagaron al maestro carpintero Diego de Morales, 20 reales por la realización de una puerta y $26 \mathrm{I}$ reales y medio al albañil Pedro Hernández, A.H.M.C. Obras y Urbanismo. Cuentas. Legajo 902.

8. A.H.M.C. Actas Capitulares. Libro 47. El escrito fue presentado el día 8 de febrero del citado año.

9. En sendos cabildos celebrados los días 3 y 7 de marzo de I608, se insistía en la necesidad de culminar las labores, A.H.M.C. Actas Capitulares. Libro 47.

10. HERNÁNDEZ DÍAZ, José; SANCHO CORBACHO, Antonio; COLLANTES DE TERÁN, Antonio: Op. cit. 274. En esta publicación se indica que se realizaron nuevas esculturas de mármol para la puerta. Sin embargo, el documento citado, así como otros hallados en el Archivo de Carmona, sólo nos habla de una intervención restauradora sobre las dos esculturas existentes en la puerta, A.H.M.C. Actas Capitulares. Libro 47. Cabildo de 29 de marzo de 1608.

II. A.H.M.C. Obras y Urbanismo. Cuentas. Legajo 902.

12. CARO, Rodrigo: Antigüedades y Principado de la llustrísima ciudad de Sevilla y Chorografia en su convento juridico o antigua Chancilleria. Sevilla, I 634, folio 156 vto. Caro recoge: "en la puerta que llaman de Cordova ay dos estatuas togadas enteras".
13. A.H.M.C. Obras y Urbanismo. Cuentas. Cuenta de la obra de la puerta de Córdoba. Legajo 902. Por los escudos de piedra percibió Salvador Rodríguez el 2 de junio de 1608, 880 reales.

14. La inscripción se ha tomado de HERNÁNDEZ DÍAZ, José; SANCHO CORBACHO, Antonio; COLLANTES DE TERÁN, Antonio: Op. cit. p. 213. Su transcripción la ofrece "El curioso carmonense ocioso divertido" (autor de un Ms. titulado Historia de la Antiquisima, muy noble y muy leal ciudad de Carmona, cuyo prólogo está concluido el 29 de septiembre de 1787). Ed. 1997, p. 112.

15. En la quinta frase de la lápida, parece que se quiso incorporar lo siguiente: "FILIPO REX III REPARAVIT". las confusiones de las letras impide la lectura correcta del texto.

16. A.H.M.C. Actas Capitulares. Libro 48. Cabildo celebrado el día 25 de febrero.

17. HERNÁNDEZ DÍAZ, José; SANCHO CORBACHO, Antonio; COLLANTES DE TERÁN, Antonio: Op. cit. pp. 213 y 274, y A.H.M.C. Actas Capitulares. Libro 71.

18. Además de la Puerta de Córdoba, la de Santa Ana ofrecía un estado similar. HERNÁNDEZ DÍAZ, José; SANCHO CORBACHO, Antonio; COLLANTES DE TERÁN, Antonio: Op. cit. p. 274.

19. JIMÉNEZ, Alfonso: La Puerta de Sevilla en Carmona, Sevilla, 1989, p. 85. La propuesta del diputado don Diego Luis de Rueda fue presentada en el cabildo celebrado el día 10 de septiembre. A.H.M.C. Actas Capitulares. Libro III. Folios I 12 vto- 113.

20. CARMONA. "Planos y pefiles semexan / tes proporcionales de la Cal / zada que sale desta Ciud. de / Carmona, y Puerta de Cor / dova en donde empieza, y / Puente en donde remata". Año de 1774, por Gregorio González y Gaspar Peña. Firmado "Gregorio González y Gaspar Peña". Escala en varas. $550 \times 1295 \mathrm{~mm}$. Tinta y aguada de varios colores, con explicación. A.H.M.C. Obras y Urbanismo. Legajo 897.

21. CARMONA. "Plan y perfiles de las obras de albañileria proyectadas en la Ciudad de Carmona execu / tado por Dn Ignacio moreno mtro mayor por su Md delos Rs Alcazares de la Ciudad / de Sevilla de la RI Universidad de dicha Ciudad y Alcalde Alarife que ha sido en ella". Año de 1775, por Ignacio Moreno. Firmado "Ignacio Moreno". Escala en varas. $1067 \times$ $2323 \mathrm{~mm}$. Tinta y aguada de varios colores, con explicación. A.H.M.C. Obras y Urbanismo. Legajo 897. El proyecto global fue valorado por Moreno en 555.230 reales, presupuestando para la puerta 16.600 reales. Este plano al que se alude en esta nota y en la anterior fue publicado por Hernández Díaz, José; Sancho Corbacho, Antonio; Collantes de Terán, Francisco. Op. cit, fig. 443 (nota 20) y fig. 444 (nota 2I).

22. A.H.M.C. Obras y Urbanismo. Planos. Proyectos y expedientes de obras. Legajo 910.

23. A.H.M.C. Actas Capitulares. Legajo 227. 
24. HERNÁNDEZ DÍAZ, José; SANCHO CORBACHO, Antonio; COLLANTES DE TERÁN, Antonio: Op. cit. p. 274. Esta noticia fue publicada de modo resumido en la citada publicación, si bien el documento recoge mayor información sobre lo que sería el inicio del proceso constructivo de la puerta. A.H.M.C. Actas Capitulares. Libro 213.

25. A.H.M.C. Actas Capitulares. Libro 214. El acuerdo fue adoptado en el cabildo celebrado el 8 de febrero. El coste de las labores ascendía a 36.200 reales

26. HERNÁNDEZ DÍAZ, José; SANCHO CORBACHO, Antonio; COLLANTES DE TERÁN, Antonio: Op. cit. pp. 213 y 274, y A.H.M.C. Actas Capitulares. Libro 219.

27. A.H.M.C. Obras y Urbanismo. Cuentas. Legajo 905.

28. Para la vida y obra de Echamorro puede consultarse, SUÁREZ GARMENDIA, José Manuel: El urbanismo en Sevilla en el siglo XIX, Sevilla, 1987, pp. 4I-44 y SANCHO CORBA$\mathrm{CHO}$, Antonio: Arquitectura barroca sevillana del siglo XVIII, Madrid, 1952, pp. 259-26I.

29. Al citado concurso se presentaron Vicente de San Martín, Francisco de Escacena, Fernando de Rosales, Antonio Balcárcel, Ginés de San Martín, Antonio de Figueroa y Antonio Márquez. Archivo Histórico Municipal de Sevilla. (A.H.M.S.). Sección V, Escribanías de Cabildo. Tomo 25. Documento I8.

30. SANCHO CORBACHO, Antonio: Op. Cit. p. 259.

31. SUÁREZ GARMENDIA, José Manuel: Op. cit. p. 42. Suárez Garmendia ha puesto este hecho de relieve, señalando como el Secretario General de la Real Academia de San Fernando, don Martín Fernández de Navarrete, en un oficio dirigido al cabildo sevillano el 4 de mayo de $|8| 8$, indicaba que no constaba como aprobado en ninguna de las Reales Academias. Esta circunstancia le produjo que precisamente la Academia le abriese expediente y le pusiese una multa de 100 ducados.

32. Ibídem. p. 260.

33. Ibídem. p. 43.

34. El templo de San Pedro de Peñaflor fue trazado por Antonio de Figueroa y desde 1788 continuado por Echamorro, LUNA FERNANDEZ-ARAMBURU, Rocío; SERRANO BARBERAN, Concepción: Planos y dibujos del Archivo de la Catedral de SeviIla. Sevilla, 1986, p. 71.

35. HERNÁNDEZ DÍAZ, José; SANCHO CORBACHO, Antonio; COLLANTES DE TERÁN, Antonio: Op. Cit. pp. 213 y 274. Y SANCHO CORBACHO, Antonio: Op. cit. P. 259.
36. Ambos diseños fueron publicados por SANCHO CORBACHO, Antonio: Op. cit. Láminas 372 y 373.

37. JIMÉNEZ MAQUEDA, Daniel: Op. cit. pp. 32 y 43.

38. VILELA GALLEGO, Pilar: "San Bartolomé de Sevilla", Archivo Hispalense, núm 221, Sevilla, 1990, p. 180.

39. Esta obra le fue atribuida por Sancho Corbacho, si bien ha sido Suárez Garmendía quien documentalmente ha podido demostrar la relación del arquitecto con la iglesia utrerana. SUÁREZ GARMENDÍA, José Manuel: Op. cit. P. 43.

40. Barcenilla realizó el proyecto en 1796, iniciándose las obras en 1800 y concluyéndose en I84I, SANCHO CORBACHO, Antonio: Op. cit. P. 26I.

41. MARTÍNEZ MONTIEL, Luis Francisco: "De Monasterio a cuartel: la fortificación de la Cartuja de Sevilla durante la Guerra de la Independencia", Archivo Hispalense, núm. 238, Sevilla, 1995, pp. 137-149.

42. FALCÓN MÁRQUEZ, Teodoro: "La arquitectura en la Baja Andalucía", Historia del arte en Andalucía, Tomo VI, Sevilla, 1994, p. 347.

43. La iglesia de Utrera le fue atribuida por SANCHO CORBACHO, Antonio: Op. Cit. P. 26I. Respecto a la iglesia gaditana véase, BANDA Y VARGAS, Antonio de la: "De la llustración a nuestros días", Historia del arte en Andalucía, Vol. VIII, Sevilla, 1994, p. 80.

44. BANDA Y VARGAS, Antonio de la: Op. cit. p. 80.

45. Esta puerta fue proyectada por el arquitecto Sebastián Van Der Borcht, en torno a 1760, aunque sólo debió realizar la fachada interior, en la que también empleó el orden jónico. Suárez Garmendia atribuye la fachada exterior a Echamorro, por las similitudes estilísticas que ofrece con la carmonense Puerta de Córdoba. SUÁREZ GARMENDIA, José Manuel: Op. cit. p. 177.

46. La imagen de la puerta es un grabado que realizó Bartolomé Tovar en 1878, probablemente para la obra de F. De Borja Palomo. SANCHO CORBACHO, Antonio: Iconografía de Sevilla, Sevilla, 1975. Lám. 136. 